\title{
Evaluación de la Atención Primaria de Salud en un contexto urbano: percepción de actores involucrados - Bañado Sur - Paraguay, 2011
}

Primary Health Care assessment in a urban context: perceptions of social actors - Bañado Sur-Paraguay, 2011

María Isabel Rodríguez-Riveros', Ivani Bursztyn², Mónica Ruoti³, Roberto Dullak4, Malvina Páez

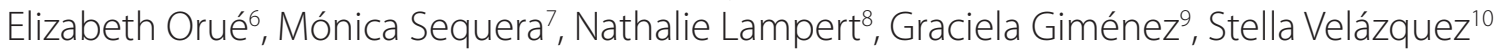

'Docente Investigadora del Instituto de Investigaciones en Ciencias de la Salud - Universidad Nacional de Asunción, Magister en Ciencias de la Educación con Especialidad en Investigación Socioeducativ Coordinadora en Paraguay del Proyecto Multicéntrico en Atención Primaria de Salu
(Segunda Etapa): Coordinadora Nacional de la Red de Investigaciones en Sistemas Servicios de Salud Nodo - Paraguay. rodriguez_riveros@hotmail.com

2 Doctor; Professor Adjunto da Faculdade de Medicina da Universidade Federal do Rio de Janeiro (UFRJ) - Rio de Janeiro (RJ), Brasil. ivani@iesc.ufrj.br ${ }^{3}$ Magister en Trabajo Social; Docente
Investigadora del Instituto de Investigaciones en Ciencias de la Salud - Universidad Nacional de Asuncion; Miembro de la Red de Salud Nodo - Paraguay. monicaruoti@tigo.com.py

${ }^{4}$ Médico Cirujano, Docente Cátedra de Epidemiología Facultad de Ciencias Médicas - Universidad Nacional de Asunción. Miembro de la Red de Investigación en e Salud Nodo -

${ }^{5}$ Magister en Salud Pública - Especialidad en Epidemiologia, Medica Clinica; Docente Investigadora en el Instituto de Investigaciones en Ciencias de la Salud - Universidad Nacional de Asunción; Vicecoordinadora de la Red de Investigaciones

Paraguay. paezmalvina@yahoo.es

${ }^{6}$ Trabajadora Social; Docente Investigadora del Instituto de Investigaciones en Ciencias de la Salud - Universidad Nacional de Asunción; Miembro de la Red de Investigaciones en Sistemas y Servicios de Salud Nodo - Paraguay.

lichiorue@hotmail.com

${ }^{7}$ Psicóloga; Docente Investigadora del Instituto de Investigaciones en Ciencias de la Salud - Universidad Nacional de Asunción Miembro de la Red de Investigaciones

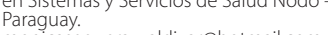

(a)

Psicóloga; Docente Investigadora de Instituto de Investigaciones en Ciencias de la Salud - Universidad Nacional de Asuncion: Miembro de la Red de Investigaciones en Sistemas y Servicios de Salud Nodo Paraguay.

nati_lampert@hotmail.com

${ }^{9}$ Bioquímica Clínica; Docente Investigadora del Instituto de Investigaciones en Ciencias de la Salud - Universidad Nacional de Asuncion; Miembro de la Red de Salud Nodo - Paraguay. gachegim@hotmail.com

${ }^{10}$ Médica; Especialista en Salud Pública, Ex-coordinadora de Atención Primaria de Salud - Ministerio de Salud Publica y Bienestar Social - Asunción, Paraguay. stell
RESUMEN En Paraguay, desde 2008 se procesa la reforma de la Atención Primaria de Salud, buscando reducir inequidades. Con el objetivo de conocer el rol de la Atención Primaria de Salud en la reordenación del sistema de salud, se utilizó la herramienta Primary Care Assessment Tool para estudiar los Equipos de Salud Familiar en Asunción. Fueron entrevistados 360 usuarios y 36 profesionales. Los resultados cubren las dimensiones acceso, puerta de entrada, vínculo, integralidad, coordinación, enfoque familiar, orientación a la comunidad, y formación profesional. Se observa que usuarios y profesionales tienen percepciones diferentes, pero convergen que el acceso es un punto débil y la formación profesional uno fuerte. Se concluye que hay una importante inversión en la Atención Primaria de Salud, pero la población aún no se está apropiando de toda su potencialidad.

PALAVRAS-CHAVE: Atención Primaria de Salud; Salud de la familia; Paraguay.

\begin{abstract}
In Paraguay, since 2008 a Primary Health Care reform is taking place, aiming at reducing inequities. With the objective of understanding the role of Primary Health Care in the reorganization of the health system, the Primary Care Assessment Tool was used to study the Family Health Teams in Asunción. We interviewed 360 users and 36 health professionals. The results cover the dimensions of access, gateway, longitudinality, portfolio, coordination, family focus, community orientation, and professional training. It was observed that users and professionals have different perceptions, but agree that access is a weakness and training a strength. It is concluded that there is an important investment in Primary Health Care, however the population is still adapting their full potential.
\end{abstract}

KEYWORDS: Primary Health Care; Family health; Paraguay. 


\section{Introducción}

Se reconoce que países que cuentan con un sistema sanitario orientado hacia la Atención Primaria de Salud (APS) tienen mejores resultados sobre la salud de la población y son más eficientes (STARFIELD, 2001), pero, según algunos autores, hay una escasa evidencia de la relación entre cada uno de los conceptos que definen la APS y la salud o la satisfacción de los ciudadanos (SANS-CORRALES et al., 2006).

Starfield et al. (2005), en el análisis de la APS, refieren una alta correlación con las características de la política, o sea la adecuada prestación de los servicios de APS estuvo asociada con el soporte de las políticas gubernamentales. Un segundo hallazgo es que el grupo de países con puntuaciones bajas en la atención primaria tenían resultados de salud más pobres y notables en los indicadores de la niñez temprana, particularmente bajo peso al nacimiento y mortalidad postneonatal (STARFIELD et al., 2005). En una revisión de las políticas de APS en la América Latina y el Caribe, fueron analizados aspectos de la APS en el contexto de su teoría y aplicación, considerando a la multidisciplinariedad como una condición básica. Todos los objetivos de la APS fueron considerados relevantes y pertinentes, siendo los de acceso universal y participación comunitaria identificados como los de mayor prioridad y el de acceso de primer contacto y trabajo intersectorial como los menos prioritarios (YAVICH et al., 2010).

La coexistencia de percepciones divergentes en la forma de interpretar el concepto de APS desarrollado en la conferencia de Alma Ata ha generado discrepancias a la hora de la aplicación de esta política (OPS, 2002). Hoy, sin embargo, existe un consenso internacional sobre las dimensiones que componen la APS. Las definiciones han evolucionado desde modelos eficientes basados en planteamientos exclusivamente técnicos, a otros que aportan una visión más holística y plural (ESCUDERO-ESCORZA, 2003; MERCADO-MARTÍNEZ et al., 2000). Esos nuevos enfoques obligan a definir la APS no sólo de la perspectiva de profesionales y gestores, sino incorporando también la perspectiva de los ciudadanos.

Varias son las razones que justifican la adopción de una estrategia renovada en cuanto a la APS: los nuevos desafíos epidemiológicos que se deben asumir; la necesidad de corregir las debilidades e incoherencias presentes en algunos de los diferentes enfoques respecto a la APS; el desarrollo de nuevos conocimientos e instrumentos sobre mejores prácticas y su contribución en la mejora de la efectividad de la APS; y, por último, el reconocimiento creciente de que la APS es una estrategia para fortalecer la capacidad de la sociedad en reducir las inequidades en salud (FRENK, 2009). Y más, se considera que un enfoque renovado de la APS es una condición esencial para cumplir con los compromisos internacionales vinculados a objetivos de desarrollo, incluidos los presentes en la Declaración del Milenio, así como para abordar los determinantes sociales de la salud, y alcanzar el nivel de salud más alto posible para todos (OPS, 2007).

La protección de la salud en Paraguay es incompleta. El sistema de salud está segmentado. Parte de la población de Asunción (27,3\%) es cubierta por la seguridad social, pero la mayoría es atendida por el sector público cuyo principal representante es el propio $\mathrm{Mi}$ nisterio de Salud; y otro segmento de relativa importancia es atendido por el sector privado. La fragmentación en estos subsectores condiciona que una parte considerable de la población (cerca de 40\%) no tenga acceso a los servicios de salud (DGEEC, 2007).

Hay evidencias de los procesos de incorporación de algunos de los principios de la APS en las políticas sanitarias de los últimos 20 años, y la transición de un nuevo gobierno instaurado en el 2008, con énfasis en la salud como un derecho fundamental, donde se establece la estrategia de priorizar a la APS, a través de la instalación de Unidades de Salud de la Familia (USF) en todo el Paraguay, como puerta de entrada al sistema público de salud, se constituye uno de los programas emblemáticos dentro de la propuesta de Política Pública para el Desarrollo Social, Paraguay para Todos y Todas como puerta de entrada al sistema público de salud (DULLAK et al., 2011).

Entre las principales fortalezas se destacan la actual condición política favorable, los recursos humanos comprometidos, las personas y organizaciones que forman parte de las comunidades como un apoyo fuerte para el desarrollo de la APS, además el apoyo internacional que se está teniendo (DULLAK et al., 2011). 
Este estudio buscó conocer el desempeño de la APS a partir de las opiniones de los diferentes actores involucrados en los servicios, con el objetivo de evaluar su rol en el reordenamiento del sistema de salud en Paraguay.

\section{Materiales y Métodos}

En este trabajo se estudiaron las USF recién instaladas en Paraguay como parte de la segunda fase de una investigación conducida en Paraguay, Argentina, Brasil y Uruguay para conocer el rol estratégico de la APS en las reformas recientes de los sistemas de salud en estos países (BURSZTYN, 2010; HEIMANN, 2011; SOLLAZZO y BERTERRETCHE, 2011; STOLKINER et al., 2011; DULLAK et al., 2011). Se eligió la Zona del Bañado Sur, en la periferia de Asunción, donde el Ministerio de Salud Pública y Bienestar Social ha instalado desde el 2008 las primeras USF. En el 2011, esta zona contaba con siete USF en las cuales actúan 12
Equipos de Salud de la Familia (ESF) conformados por un médico/a general o especialista en medicina familiar, uno licenciada/o en enfermería u obstetricia, un auxiliar o técnico/a en enfermería y cinco agentes comunitarios. Cada ESF tiene un territorio social definido, y es responsable del cuidado de aproximadamente 700 familias, con una cobertura de 3.500 a 4.000 personas. El Bañado Sur es un área urbana, compuesta por grupos con distintos niveles de ingreso y variado acceso a la infraestructura de los servicios de salud, ya sean públicos o privados.

Para el levantamiento de encuesta a los usuarios y profesionales de salud, se utilizó el Primary Care Assessment Tool (PCAT), que es una herramienta de evaluación desarrollada por la John Hopkins Primary Care Policy Centre for Undeserved Populations, y validada por Almeida y Macinko (2006). Las preguntas que evalúan el desempeño de los atributos de la APS poseen categorías de respuestas cerradas, que incluyen una escala valorativa preestablecida con las opciones demostradas en el Cuadro 1.

Tabela 1. Escores de respuestas de Evaluación de la Atención Primaria

\begin{tabular}{lcc|c|c|c|c}
\hline Escore & 1 & 2 & 3 & 4 & 5 & 6 \\
Valores & Nunca & Casi nunca & Algunas veces & Muchas veces & Casi siempre & Siempre \\
& $0 \%$ & $1-20 \%$ & $21-40 \%$ & $41-60 \%$ & $61-80 \%$ & $81-100 \%$
\end{tabular}

Las dimensiones de la APS abordadas por el instrumento son:

- Puerta de entrada: existencia de una fuente regular de atención disponible dirigida a la atención en salud de la población;

- Acceso: oportunidad de utilización de los servicios por la ausencia de barreras económicas, geográficas, organizacionales y culturales;

- Vínculo (longitudinalidade): posibilidad de lograr una atención en salud permanente en el tiempo, así como regularidad en la relación entre usuario y proveedor estable de los servicios médicos;
- Integralidad: conjunto de servicios disponibles que permitan cubrir las necesidades y problemáticas de la salud de los diversos grupos que conforman la población usuaria, también incluye a los diversos componentes de la atención en salud, o sea, promoción, prevención, curación, y rehabilitación;

- Coordinación: existencia de mecanismos de comunicación, referencia y contrarreferencia entre los diversos proveedores de servicios, para evitar la fragmentación de la atención en salud;

- Enfoque familiar: considera la importancia del entorno y los antecedentes familiares 
dentro de los condicionantes del proceso salud-enfermedad, así como la necesidad de facilitar y fomentar la participación y el apoyo familiar en la atención en salud;

- Orientación a la comunidad: fomento de la participación comunitaria en la definición de las necesidades y en las alternativas para su intervención;

- Formación profesional: recurso humano con capacitación y formación en el enfoque de APS para facilitar el cumplimiento de sus atributos.

Las preguntas y escalas han sido similares para todos/as los/as entrevistados/as (usuarios/as, profesionales de APS) a fin de facilitar la comparación de los resultados de la evaluación desde las diferentes perspectivas de actores involucrados. Los instrumentos fueron ajustados de acuerdo a las características del país.

El muestreo utilizado, no probabilístico por conveniencia, fue constituido por 360 usuarios y 36 profesionales de los 12 ESF del Bańado Sur. Para cada equipo fueron encuestados 30 usuarios en sus hogares y tres profesionales de salud.

Para la recolección de los datos, se conformó un equipo de trabajo con seis docentes investigadoras de los Departamentos de Ciencias Sociales y Salud Pública del Instituto de Investigaciones en Ciencias de la Salud de la Universidad Nacional de Asunción, que a su vez son miembros de la Red de Investigación en Sistemas y Servicios de Salud Nodo Paraguay.

Fueron buscados el consentimiento y la cooperación para desarrollar el trabajo de campo junto a la Coordinadora General de las Unidades de Salud de Asunción, y el Director de la XVIII Región Sanitaria de la Capital, del Ministerio de la Salud Pública y Bienestar Social. Se procedió de igual forma con los responsables de cada USF. El reconocimiento de la zona se hizo con apoyo de los agentes comunitarios de salud correspondientes.

El trabajo de campo se concluyó en cuatro meses, entre abril y julio de 2011, con dificultades por la inclemencia del tiempo, ya que es una zona inaccesible e intransitable en tiempos lluviosos. En relación a los profesionales de los equipos, fue difícil acordar las entrevistas por el exceso de pacientes que deben ser atendidos por día, las visitas domiciliarias que deben realizar $y$, en el caso de los agentes, estos pasan la mayor parte del tiempo en sus respectivas comunidades.

Se efectuó un análisis estadístico descriptivo (distribuciones de frecuencias, medias aritméticas de las variables de cada dimensión), se construyeron los índices correspondientes por dimensión y el compuesto global.

El proyecto obtuvo aprobación de los Comités Científico y Ético del Instituto de Investigaciones en Ciencias de la Salud de la Universidad Nacional de Asunción. Fueron encuestados las personas que aceptaron voluntariamente participar del estudio, previa explicación de los objetivos del mismo. Los cuestionarios fueron anónimos y se guardó rigurosa confidencialidad de la información obtenida.

\section{Resultados}

Entre las características demográficas de los usuarios entrevistados, se encuentra una edad media de 41 años (desviación estándar \pm 18 años) siendo la mínima de 14 y la máxima de 90 ańos, $83 \%$ del sexo femenino y $17 \%$ del masculino. En cuanto a la escolaridad, el $8 \%$ son analfabetos, $29 \%$ cuentan con primaria completa, y el $21 \%$ incompleta; $14 \%$ con secundaria completa y $23 \%$ incompleta; el $2 \%$ cuenta con estudios superiores concluidos, y el 3\% no concluyó.

$\mathrm{Al}$ respecto a los servicios básicos y al nivel de consumo, casi el $100 \%$ cuentan con luz eléctrica, seguido de provisión de agua, tenencia de televisor, heladera y celular. En menor proporción con servicios sanitarios dentro de la casa y lavarropas; y muy pocos cuentan con horno microondas y automóvil.

$\mathrm{Al}$ indagar sobre la percepción de su salud en los últimos 30 días, el $50 \%$ consideraron bueno a muy bueno; mientras que la otra mitad consideró de regular a malo y muy malo. El 23\% dejó de realizar sus actividades por problemas de salud, con una media de nove días. Tres personas se encontraban sin actividad laboral desde hace dos meses, dos y diez años, respectivamente. Por otro lado, el 56\% reconoció haber padecido enfermedades en los últimos 30 días, siendo en orden de 
frecuencia, de la columna o dolor de espalda (52\%); hipertensión arterial (52\%); en menor proporción: diabetes $(18 \%)$, artritis o reumatismo (17\%), bronquitis o asma (12\%), enfermedades del corazón (10\%), cáncer, y depresión (2\%).

Los miembros del equipo de salud entrevistados fueron en su mayoría profesionales de la salud (médicos, enfermeras, obstetras y odontóloga); en menor proporción los agentes comunitarios.

Comparando la manera como los usuarios y profesionales evalúan las dimensiones estudiadas de la APS, se observa que en general los usuarios tienen una percepción más negativa que los profesionales. Dado que los usuarios fueron entrevistados en su hogar, esto puede haber contribuido a que se sientan cómodos para responder con más críticas. Por el lado de los profesionales, hay, en este momento, una gran inversión para especializarse y potenciar los médicos de familia, que puede haber contribuido a una evaluación más positiva. Un tercer aspecto podría estar relacionado con la experiencia reciente de los usuarios, una vez que la estrategia de salud de la familia es relativamente nueva, ellos aún están en proceso de conocerla. Como fortalezas, ambos, usuarios y profesionales, reconocen el entrenamiento profesional, vínculo con el servicio, orientación a la comunidad y puerta de entrada. Como debilidad hay acuerdo en cuanto al acceso (Gráfico 1).

Grafico 1. Evaluación de usuarios y profesionales cuanto a la performance de la Atención Primaria de Salud - Bañado Sur, 2011

Fuente: Elaboración propia.

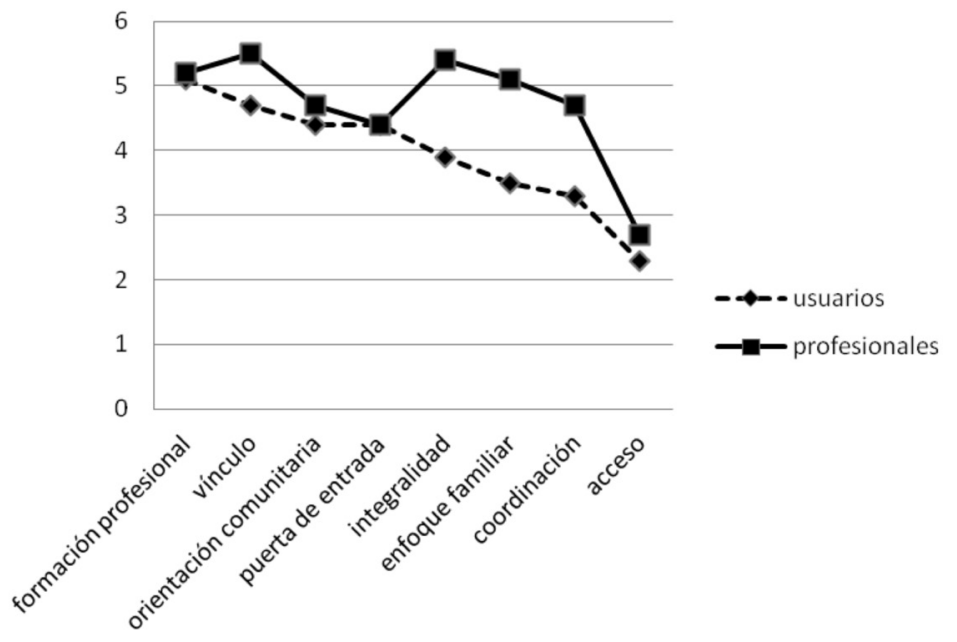

\section{Acceso}

En cuanto al acceso, hay coherencia entre usuarios y profesionales. Ambos brindaron mejores escores a la 'facilidad de obtener citas no urgentes', 'provisión de medicamentos' y 'tiempo de espera en el servicio', además de la ausencia de copago. Los peores escores fueron para la disponibilidad de contacto por teléfono para marcación de citas y restricción del horario de funcionamiento (Gráfico 2).

\section{Puerta de entrada}

Aunque los usuarios reconozcan el nivel de la salud de la familia como útil, sea para los cuidados preventivos o para el tratamiento de los problemas, ellos afirman que el contacto con especialistas no requiere la derivación del profesional de esto nivel. Los profesionales presentaron escores altos para esta dimensión, incluso para el ítem relativo a la necesidad de contacto con el primer nivel antes de consultar con especialista. 
Grafico 2. Evaluación de usuarios y profesionales cuanto al acceso a la Atención Primaria de Salud - Bañado Sur, 2011

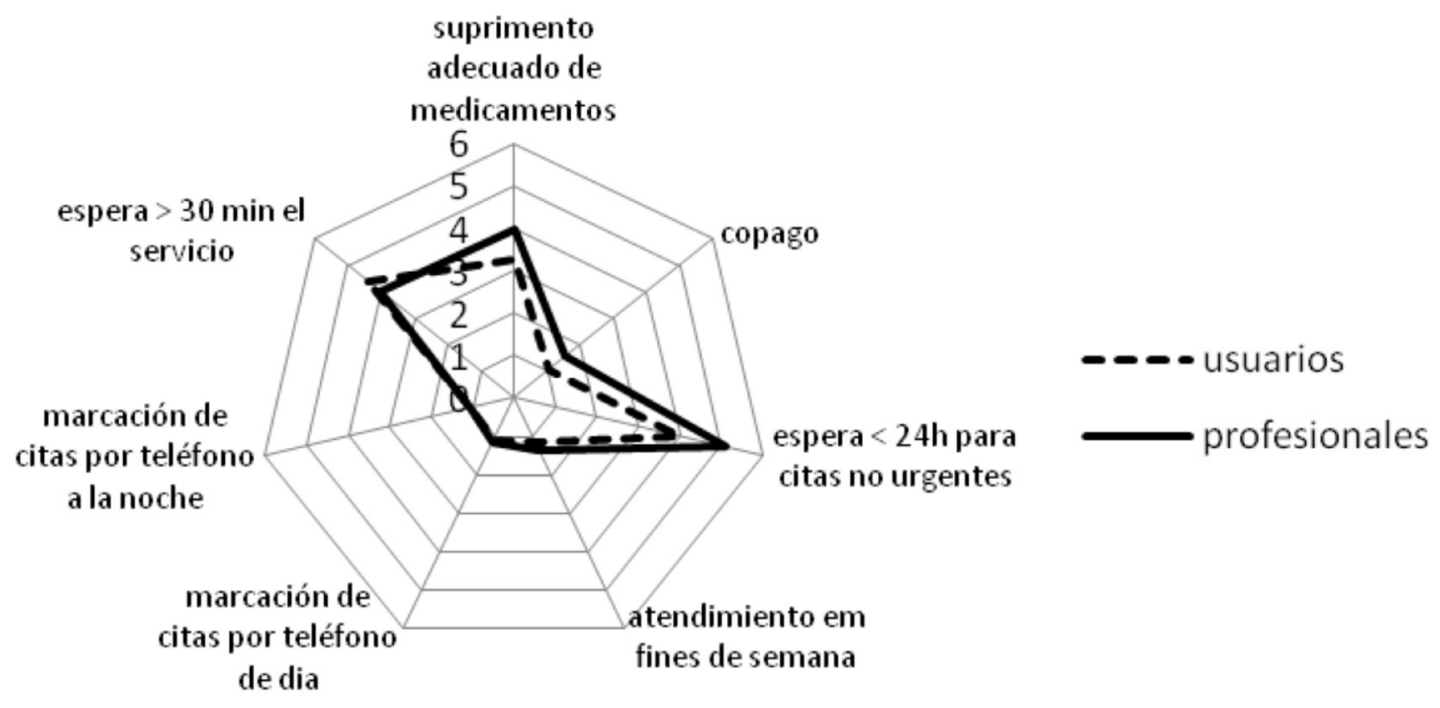

Fuente: Elaboración propia.

Grafico 3. Evaluación de usuarios y profesionales cuanto a lo vinculo en la Atención Primaria de Salud - Bañado Sur, 2011

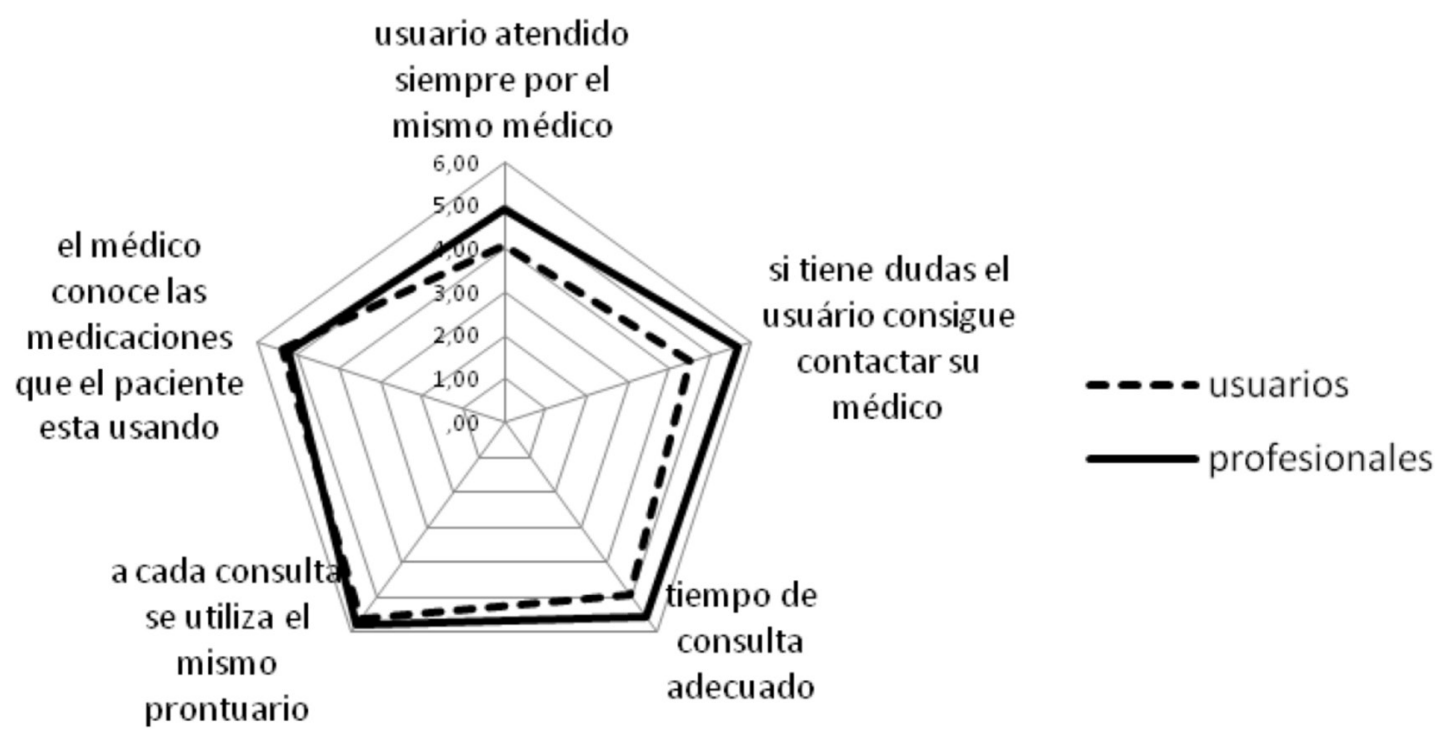




\section{Vínculo (longitudinalidade)}

La visión de los usuarios y profesionales es similar en cuanto al vínculo. Aunque ambos hayan evaluado esta dimensión positivamente, se observa que los profesionales presentan escores más altos (Gráfico 3).

\section{Integralidad (portafolio)}

La percepción respecto al portafolio de servicios varía significativamente entre usuarios y profesionales. Probablemente la vacunación, el cuidado con los niños, el pre-natal, el planeamiento familiar, la atención a los problemas crónicos y epidémicos, el tratamiento de heridas y el cuidado odontológico sean los servicios más utilizados y, consecuentemente, los más bien reconocidos por la población de usuarios. Las actividades educativas son las menos percibidas por los mismos (Gráfico 4).

\section{Coordinación}

En la dimensión de la coordinación hay mucha discrepancia entre la evaluación de usuarios y profesionales, excepto por la utilización de la libreta de la embarazada, bien evaluada por ambos y la existencia de un registro pre escrito de contrarreferencia, cuya evaluación fue mala por ambos. Probablemente, la percepción de los usuarios está influenciada por el hecho de que la mayoría de los casos maneja el médico de familia, sin utilizar recursos extras como teste de diagnósticos, exámenes radiológicos, o consultas con especialistas de niveles más complejos (Gráfico 5).

Grafico 4. Evaluación de usuarios y profesionales cuanto a la coordinación en la Atención Primaria de Salud - Bañado Sur, 2011

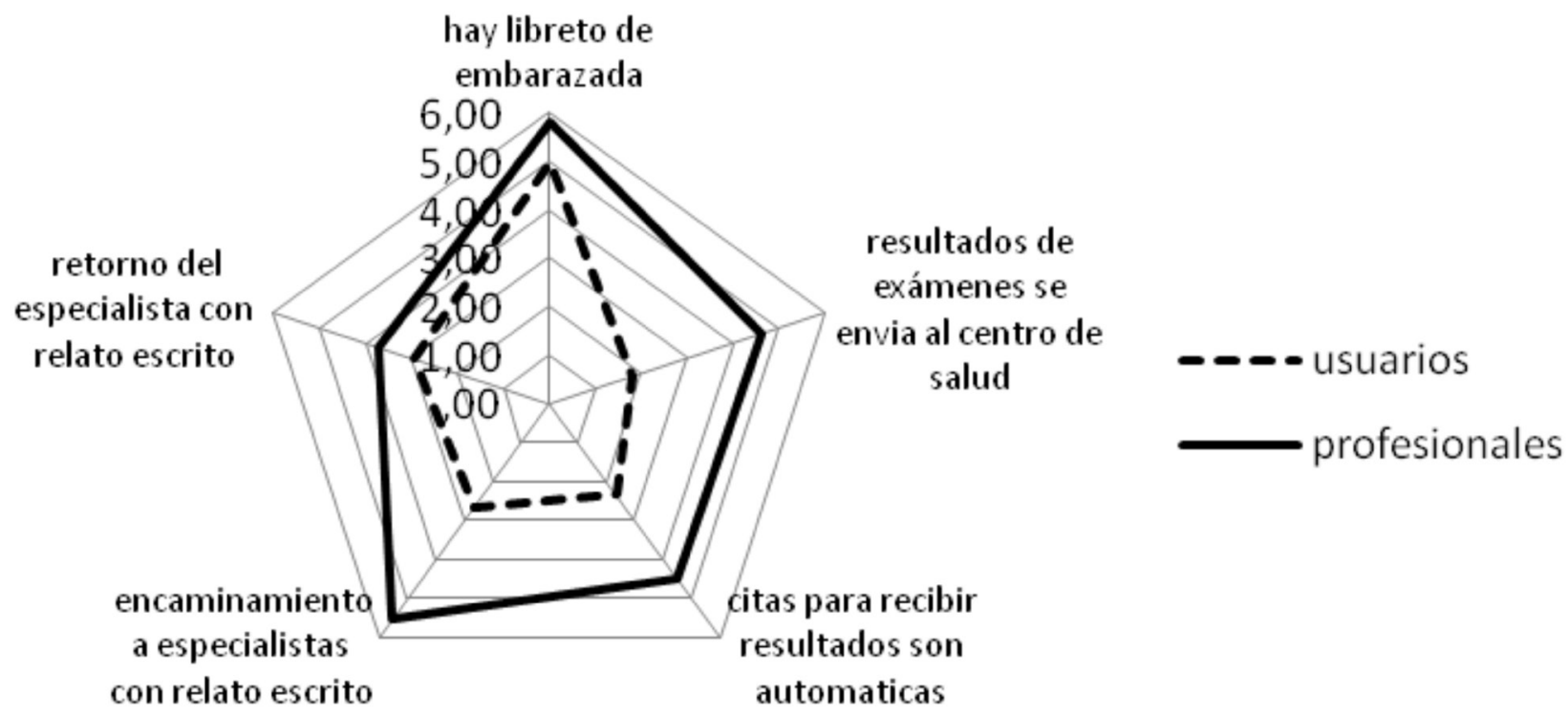


Grafico 5. Evaluación de usuarios y profesionales cuanto al enfoque familiar de la Atención Primaria de Salud - Bañado Sur, 2011

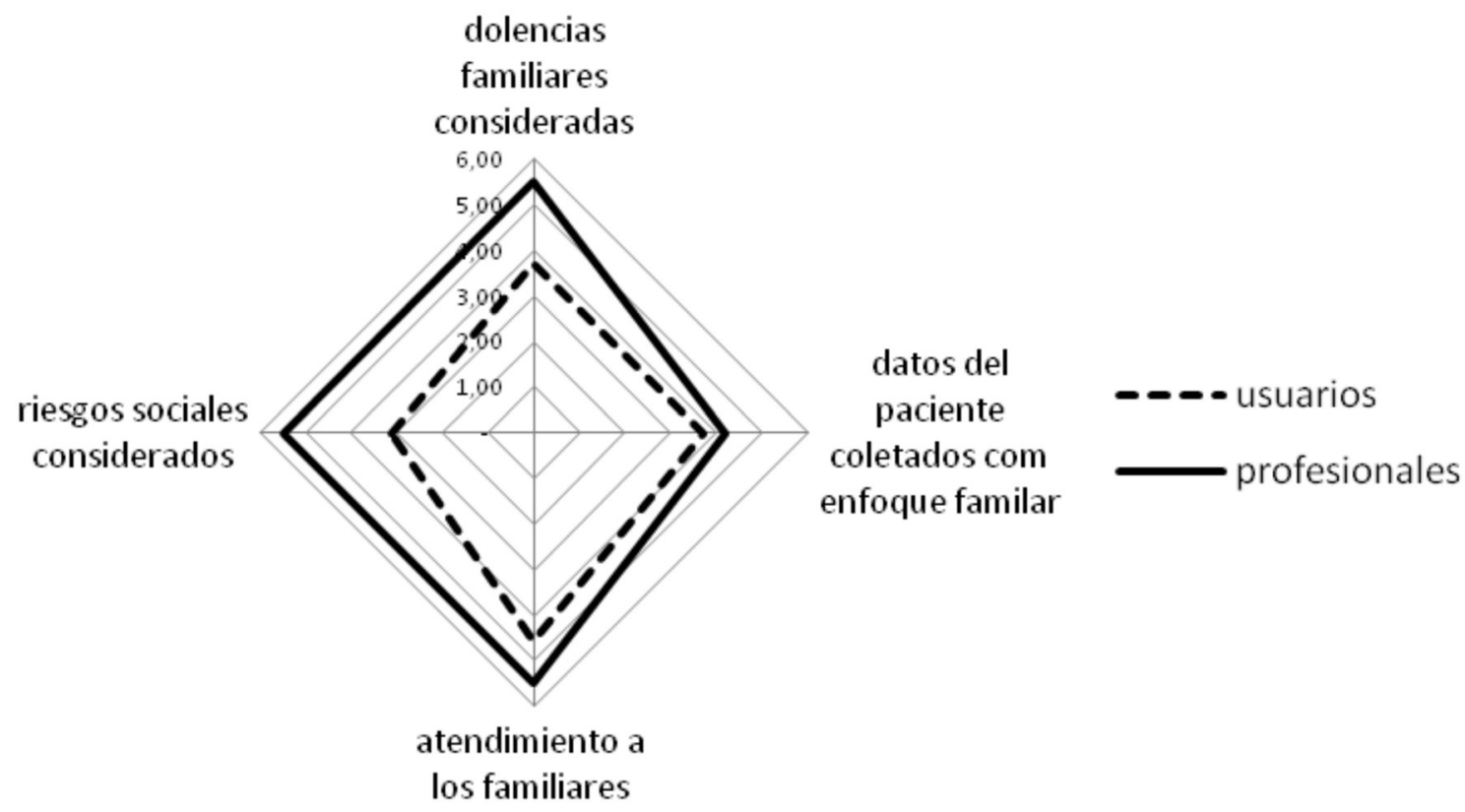

Fonte: Elaboração Própria

Grafico 6. Evaluación de usuarios y profesionales cuanto al enfoque familiar de la Atención Primaria de Salud - Bañado Sur, 2011

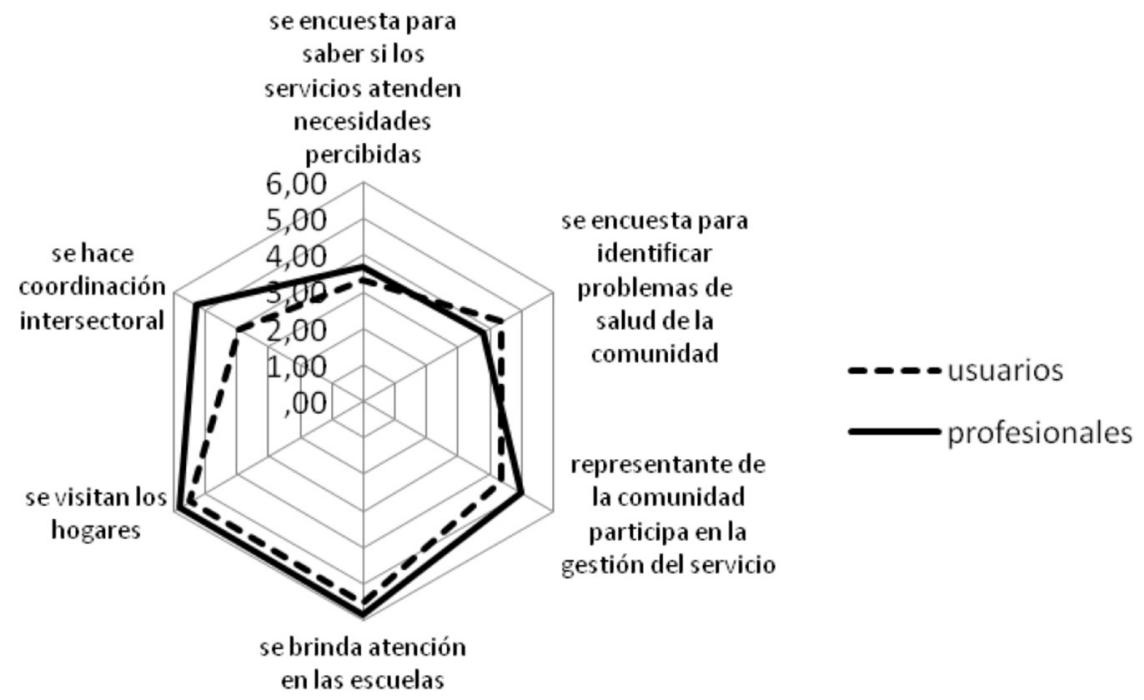




\section{Enfoque familiar}

Los usuarios evalúan el enfoque familiar como deficiente, no perciben que las dolencias familiares y los riesgos sociales son considerados. Contrariamente, los profesionales brindan altos escores para estos dos ítems (Gráfico 6).

\section{Orientación a la comunidad}

En esta dimensión hay una concordancia considerable entre usuarios y profesionales. Para ambos los servicios extramurales, tales como visitas a los hogares y servicios en las escuelas, ellos son los mejores evaluados. El peor escore recibió la participación comunitaria (Gráfico 7).

\section{Formación profesional}

La formación profesional está bien evaluada por ambos los usuarios y los profesionales. Los usuarios reconocen que existe al menos un médico y una licenciada en enfermería en los ESF, que los profesionales les tratan bien y resuelven sus problemas y, además, revelan que recomendarían estos servicios a otras personas.

Los profesionales confirman que en los ESF hay por lo menos un médico y opinan que los licenciados no remplazan a estos, que los médicos reciben formación en APS así como otros profesionales también y, además, el equipo estás preparado para el abordaje multicultural.

\section{Discusión y consideraciones finales}

La implementación de un sistema de salud basado en APS constituye un componente emblemático del programa de gobierno de 2008 al 2013. Con la mirada puesta en los principios, fundamentos y filosofía de la APS renovada, se plantea un sistema público que garantice la cobertura y el acceso universal a los servicios, los cuales sean aceptables para la población y promuevan la equidad. A partir del 2008 se organizaron e implementaron las USF como una estrategia operativa; se instalaron en el área periurbana de la capital del país extendiéndose luego a comunidades del interior, zonas urbanas y rurales, constituyendo los territorios sociales; hasta llegar a un total de 704 en enero de 2012. Las USF se constituyeron en la puerta de entrada al sistema y buscan integrarse al sistema público de salud por medio de la Red Integral de Servicios de Salud (RISS).

El estudio encontró diferencias en la percepción de usuarios y profesionales en cuanto a las dimensiones estudiadas. En ambos actores, hay una coincidencia en la dimensión con peor (acceso) y en la de mejor desempeño (formación profesional). La evaluación de los profesionales es claramente más positiva y probablemente refleja la gran inversión que se está haciendo en la APS.

En la dimensión acceso, al desagregar y analizar cada ítem individualmente se visualiza que los cuatro con baja puntuación están relacionados con aspectos que no hacen parte del diseño o modelo de funcionamiento de las USF implementadas desde el 2008; más bien éstas se relacionan con la promoción de la salud, prevención de las enfermedades y rehabilitación, cuyo ESF funciona 40 horas semanales, de lunes a viernes con horario establecido de las 07 a las 15 horas. Es importante considerar la necesidad de incorporar ajustes en el modelo; teniendo en cuenta principalmente la realidad local de trabajadoras y trabajadores, que debido a la naturaleza de sus actividades laborales, sólo se encuentran en sus hogares en la tarde/noche o en los fines de semana. Por otro lado, se refleja la necesidad de recordar y/o divulgar en la comunidad y a los usuarios del cómo y para qué funcionan las USF, las cuales están completamente establecidas en el Manual de Funcionamiento de las USF.

En el análisis de la dimensión puerta de entrada, tanto los profesionales como los usuarios han identificado satisfactoriamente a la APS como puerta de entrada, en base a los indicadores estudiados. Es importante señalar que aunque exista este reconocimiento, se observa la fragilidad del sistema, pues los usuarios en general reconocen que no necesitan realizar consulta previa en su USF antes de recurrir con un especialista.

La dimensión vínculo (longitudinalidade) presentó una alta valoración tanto por los usuarios como por los profesionales, lo que indica un buen desempeño. En las USF se concibe una mayor familiaridad entre 
los miembros del ESF, los usuarios y la comunidad, el trato entre los mismos es familiar, hecho por el cual el sistema favorece el establecimiento de vínculos de responsabilidad y confianza entre profesionales y familias, y permite una comprensión ampliada del proceso salud/enfermedad y de la necesidad de intervenciones a partir de los problemas y de las demandas identificadas. El vínculo genera en los usuarios un nivel de seguridad y confianza. Tener la posibilidad de ser atendido por el mismo profesional, aclarar sus dudas, interactuar e intercambiar sobre el motivo de su consulta, enfermedad o tratamiento es un valor agregado del sistema establecido en el escuchar y atender a las necesidades de los usuarios en todas las acciones, proporcionando atención humanizada y estableciendo o reforzando el vínculo del ESF con las personas (PARAGUAY; MSP; BS, 2010).

Contar con una población adscripta favorece la relación del ESF y los usuarios, además, el vínculo de la población con la USF requiere el establecimiento de fuertes lazos interpersonales que reflejan la cooperación mutua entre las personas de la comunidad y los profesionales de la salud (PARAGUAY; MSP; BS, 2010). La esencia de la longitudinalidade es una relación que se establece a lo largo del tiempo, independientemente del tipo o de la presencia de un problema de salud, entre individuos y un profesional del equipo de salud.

$\mathrm{Al}$ analizar las respuestas del atributo integralidad (portafolio de servicios), se observó que los profesionales han identificado plenamente el portafolio de servicios que ofrecen, lo cual es muy positivo, pues refleja que conocen la amplia gama de servicios disponibles a los usuarios y comunidad (media de 5,45). En contraposición, los usuarios fueron capaces de identificar aquellos servicios que son tangibles y asociados directamente a la enfermedad, demostrando baja percepción a actividades de promoción y educación. Con esto, surge la necesidad de divulgar el portafolio de servicios a nivel de usuarios y comunidad, lo que posibilitará el uso de los servicios que apuntan a la mejoría de la salud de la población.

La atención integral, integrada y continua implica que el portafolio o cartera de servicios disponibles debe ser suficiente para responder a las necesidades de salud de la población, incluyendo la promoción, la prevención, lo diagnóstico precoz, la atención curativa, rehabilitadora y paliativa, y el apoyo para el autocuidado (OPS, 2007). La integralidad es una función del sistema de salud e incluye prevención, y atención primaria, secundaria, terciaria, y paliativa (OPS, 2008).

Existe una marcada discrepancia entre los profesionales y los usuarios respecto a los atributos correspondientes a la coordinación, la cual es un aspecto relevante cuando se trata de diagnosticar y acompañar problemas que necesitan de exámenes o consultas a especialistas. La baja percepción de los usuarios en contraste con los profesionales puede sugerir que gran parte de la utilización de la APS se refiere a aspectos más simples que las maneja el profesional sin necesidad de otros recursos.

Al igual que en portafolio de servicio y coordinación, en el atributo enfoque familiar se mantiene la diferencia en los escores de profesionales y usuarios. Hay una cierta coherencia entre los hallazgos que muestran baja percepción de los usuarios con relación a las actividades educativas y de promoción, y la baja percepción de que los servicios consideran los riesgos sociales. Una vez más se observa la debilidad de la APS en consolidarse de acuerdo con sus principios, y la importancia de reforzarlos.

Los hallazgos en la dimensión 'orientación a la comunidad' ayudan a comprender lo que fue expresado. Usuarios y profesionales están de acuerdo en que hay debilidades respecto a realizar encuestas de las necesidades y del grado de satisfacción de los usuarios con el servicio. Es importante fortalecer este tipo de acción con vistas a aumentar el vínculo de la población con el servicio no sólo en momentos agudos, pero también en actividades de promoción y mejoría de las condiciones de vida y salud. Todavía, es importante resaltar que los escores presentados son en general más elevados que aquellos de otros estudios similares realizados en Brasil que presentaron escores menores de tres para esta dimensión (ELIAS et al., 2006).

La concordancia entre profesionales y usuarios de que la formación profesional es un punto fuerte es un reconocimiento que los esfuerzos para capacitar los profesionales con habilidades específicas para la APS está alcanzando sus objetivos y que los usuarios están satisfechos con su atención. La mayoría recomendaría la USF 
a un amigo $(5,0)$ y considera que los profesionales de salud de esta USF se relacionan bien con su comunidad $(5,1)$.

Como conclusión, se puede afirmar que el desarrollo de la Estrategia Salud de la Familia y la implementación de las USF en Bañado Sur están produciendo cambios para la población respecto a la salud. Hay una aproximación entre el servicio y la gente, con mayor acceso a actividades preventivas y de control de la salud. La manera como los usuarios perciben los nuevos recursos está marcada por toda la experiencia y acumulo histórico en la relación usuario/servicio de salud y médico/paciente. A partir de los resultados de este estudio hay razones para creer que el tiempo permitirá que los usuarios se apropien más de las propuestas de este nuevo modelo, logrando mejor interacción y cooperación entre ellos y los ESF. Para que esto ocurra es imprescindible que los servicios de salud de la familia sean capaces de atender a los usuarios en la integralidad de sus necesidades, mismo que demanden procedimientos más complejos, sea directamente o por medio de direccionamiento a otros niveles.

\section{Agradecimientos}

Agradecemos al International Development Research Center (IDRC), a la Escuela Nacional de Salud Pública de la Fundación Oswaldo Cruz, y al Instituto de Investigaciones en Ciencias de la Salud de la Universidad Nacional de Asunción, por el apoyo a esta investigación.

\section{Referências}

ALMEIDA, C.; MACINKO, J. Validação de uma metodologia de avaliação rápida das características organizacionais e do desempenho dos serviços de atenção básica do Sistema Único de Saúde (SUS) em nível local. Brasília: Organização Pan-Americana da Saúde, 2006.

BURSZTYN, l. et al. Notas para el estudio de la Atención Primaria en contextos de sistemas de salud segmentados. Revista de Salud Pública, v. 12, suplemento 1, 2010, p. 77-88.

DIRECCIÓN GENERAL DE ESTADÍSTICAS, ENCUESTAS Y CENSOS (DGEEC). Encuesta permanente de hogares. Asunción: DGEEC, 2007.

DULLAK, R. et al. Atención Primaria en Salud en Paraguay: panorámica y perspectiva. Ciência \& Saúde Coletiva, v. 16, n. 6, 2011, p. 2741-2751.

ELIAS, P.E. et al. Atenção Básica em saúde: comparação entre PSF e UBS por estrato de exclusão social no município de São Paulo. Ciência \& Saúde Coletiva, v. 11, n. 3, 2006, p. 633-641.

ESCUDERO-ESCORZA, T. Desde los testes hasta la investigación evaluativa actual. Un siglo, el XX, de intenso desarrollo de la evaluación en educación. Revista Electrónica de Investigación y Evaluación Educativa, v. 9, n. 1, 2003, p. 11-43.

FRENK, J. Reinventing primary health care: the need for systems integration. Lancet, v. 374, 2009, p. 170-173.
HEIMANN, L.S. et al. Atenção primária em saúde: um estudo multidimensional sobre os desafios e potencialidades na Região Metropolitana de São Paulo (SP, Brasil). Ciência \& Saúde Coletiva, v. 16, n. 6, 2011, p. 2877-2888.

MERCADO-MARTÍNEZ, F.J.; RAMOS-HERRERA, I.M.; VALDEZ-CURIEL, E. La perspectiva de enfermos crónicos sobre la atención médica en Guadalajara, México. Un estudio cualitativo. Cadernos de Saúde Pública, México, n. 16, 2000, p. 759-772.

ORGANIZACIÓN PANAMERICANA DE LA SALUD; UNIVERSIDAD DE CHILE. Revisión de las políticas de atención primaria de salud en América Latina y el Caribe: informe final. Washington: OPS, 2002.

ORGANIZACIÓN PANAMERICANA DE LA SALUD. Sistemas de salud basados en la Atención Primaria de Salud: estrategias para el desarrollo de los equipos de APS. Washington,: OPS, 2008.

Renovación de la atención primaria de salud en las Américas: documento de posición de la Organización Panamericana de la Salud. Washington: OPS, 2007.

PARAGUAY. MINISTERIO DE SALUD PÚBLICA Y BIENESTAR SOCIAL. Manual de Funcionamiento de las Unidades de Salud de la Familia (USF). Asunción, Paraguay, 2010.

SANS-CORRALES, M. et al. Family medicine attributes related to satisfaction, health and costs. Family Practice, v. 23, 2006, p. 308-316. 
SOLLAZZO, A.; BERTERRETCHE, R. El Sistema Nacional Integrado de Salud en Uruguay y los desafíos para la Atención Primaria. Ciência \& Saúde Coletiva, v. 16, n. 6, 2011, p. 2829-2840.

STARFIELD, B. Atención primaria: equilibrio entre necesidades de salud, servicios y tecnología. Barcelona: Masson, 2001.

STARFIELD, B.; SHI, L.; MACINKO, J. Contribution of Primary Care to Health Systems and Health. The Milbank Quarterly, v. 83, n. 3, 2005, p. 457-502.

STOLKINER, A.; COMES, Y.; GARBUS, P. Alcances y potencialidades de la Atención Primaria de la Salud en Argentina. Ciência \& Saúde Coletiva, v. 16, n. 6, 2011, p. 2807-2816.
YAVICH, N. et al. Construyendo un marco de evaluación de la atención primaria de la salud para Latinoamérica. Salud Pública, México, v. 52, n. 1, en./feb. 2010.

Recibido para publicação em Abril/2012

Versão definitiva em Agosto/2012

Suporte financiero: Programa de investigación en Salud Global Teasdale-Corti - un programa de investigación

Conflito de interesse: inexistente 